\title{
Faddeev description of two-hole-one-particle motion and the single-particle spectral function
}

\author{
C. Barbieri and W. H. Dickhoff \\ Department of Physics, Washington University, St. Louis, Missouri 63130
}

(Received 27 October 2000; published 20 February 2001)

\begin{abstract}
The Faddeev technique is employed to address the problem of describing the influence of both particleparticle and particle-hole phonons on the single-particle self-energy. The scope of the few-body Faddeev equations is extended to describe the motion of two-hole-one-particle (two-particle-one-hole) excitations. This formalism allows one to sum both particle-particle and particle-hole phonons, obtained separately in the random phase approximation. The appearance of spurious solutions for the present application of the Faddeev method is related to the inclusion of a consistent set of diagrams. The formalism presented here appears practical for finite nuclei and achieves a simultaneous inclusion of particle-particle and particle-hole phonons to all orders while the spurious solutions are properly eliminated.
\end{abstract}

DOI: $10.1103 /$ PhysRevC.63.034313

PACS number(s): 21.60.Jz, 21.10.Jx

\section{INTRODUCTION}

In recent years, the study of $\left(e, e^{\prime} p\right)$ reactions has been one of the most useful tools to probe correlations in nuclei. Absolute spectroscopic factors have become available for many closed-shell nuclei [1-3] demonstrating that the removal probability for nucleons from these systems is reduced by about $35 \%$ in comparison with the simple shell model. The theoretical description of this reduction requires the inclusion of both short-range and long-range correlations. For nuclear matter a strength removal of about $15 \%$ is obtained by including short-range correlations [4]. For ${ }^{16} \mathrm{O}$ the inclusion of short-range correlations leads to removal of single-particle (s.p.) strength of the order of $10 \%[5,6]$. The inclusion of long-range correlations for heavier nuclei like ${ }^{48} \mathrm{Ca}$ yields a qualitative description of the s.p. strength distribution by including in the nucleon self-energy the coupling to either low-lying collective particle-hole (ph) or particle-particle (pp) phonons calculated in random phase approximation (RPA) [7]. The additional depletion of about $10 \%$ due to short-range correlations for this nucleus leads to a reasonable quantitative agreement for the largest fragments of the experimental strength distribution.

Theoretical calculations of hole spectroscopic factors for ${ }^{16} \mathrm{O}$ are not so successful. The experimental spectroscopic strength [8] for the knockout of a proton from both the $p_{1 / 2}$ and $p_{3 / 2}$ shells corresponds to about $60 \%$. The $10 \%$ reduction due to short-range correlations is mostly compensated by the proper inclusion of the center-of-mass motion that enhances the probability for $p$ removal by about 7\% [9]. Calculations based on the Green's function approach, including both long- and short-range correlations, yield about a $25 \%$ reduction [10]. These results still need to be corrected for the center-of-mass effect. It is therefore fair to conclude that the present theoretical results for ${ }^{16} \mathrm{O}$ are still about $20 \%$ away from the experimental data. The importance of lowenergy correlations is clearly demonstrated by the results of Ref. [10] and their proper inclusion is therefore crucial for a complete understanding of this puzzle. In the latter work the self-energy was obtained including the effects of interactions between both $\mathrm{pp}$ and ph excitations in the Tamm-Dancoff approximation (TDA). In order to account for the coupling to collective excitations that are actually observed in ${ }^{16} \mathrm{O}$ it is necessary to at least consider an RPA description of the isoscalar negative parity states [11]. To account for the lowlying isoscalar positive-parity states an even more complicated treatment will be required. Sizable collective effects are also present in the pp and hole-hole (hh) excitations involving tensor correlations for isoscalar and pair correlations for isovector states. Another argument to improve the description of the coupling of s.p. states to low-lying collective excitations is provided by the lack of fragmentation at low energy obtained in present theoretical studies [10] in disagreement with experimental data.

One of the goals of the present work is to account for the collectivity in the ph and $\mathrm{pp}$ (hh) channels in a consistent way while including these excitations at least at the RPA level. Since the observed fragmentation and depletion of the s.p. strength in ${ }^{16} \mathrm{O}$ is quite substantial, it is reasonable to assume that these features are also important in the description of the excitations that contribute to the self-energy. This results in a self-consistent formulation where the dressing of the nucleons is incorporated in the description of the collective excitations that ultimately lead to the dressing itself. For this reason the present work will be formulated using selfconsistent Green's functions (SCGF). This type of selfconsistency must also be considered in describing pairing correlations in semimagic nuclei $[12,13]$.

A formalism in which both pp and ph phonons are treated at the RPA level in the self-energy was proposed in [14]. This work focused on the two-time two-particle-one-hole $(2 \mathrm{p} 1 \mathrm{~h})$ propagator and generated a formulation that reduces to either including the pp-RPA or the ph-RPA phonons in the self-energy when ph or pp vertices are omitted, respectively. This expansion, however, was obtained using some drastic assumptions and disregarding some of the constraints that arise when propagators in different diagrams of the expansion are connected. Related to this issue is the appearance of unphysical solutions for the $2 \mathrm{p} 1 \mathrm{~h}$ propagator that have been discussed in [15]. Results in [15] have therefore been obtained mostly for the TDA treatment of the $2 \mathrm{p} 1 \mathrm{~h}$ propagator. Moreover, this approximation was obtained by employing mean-field (single-pole) s.p. propagators. In the present selfconsistent treatment, which sums fully dressed propagators, 

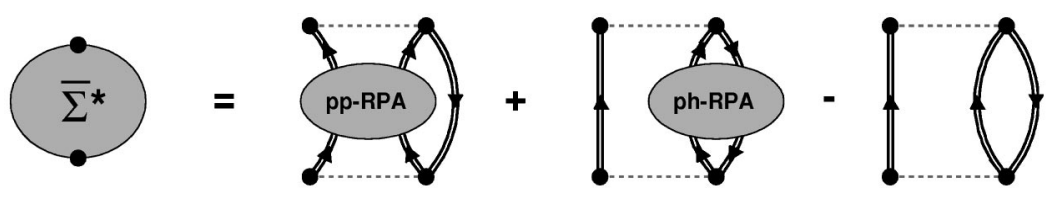

FIG. 1. Example of an approximation for the self-energy. Although this approximation contains both ph and pp correlations, it would generate incorrect results due to the need of subtracting the second order term to avoid double counting.

this approach is no longer possible.

To proceed with the inclusion of both pp and ph collectivity in the nucleon self-energy it is important to note that the naive summation of diagrams containing both pp and ph phonons leads to serious inconsistencies. This approximation is depicted in Fig. 1. The last of the three diagrams on the right-hand side is already contained in each of the other two and must therefore be subtracted to avoid double counting. This subtraction introduces spurious poles in the Lehmann representation of the self-energy and generates meaningless solutions of the Dyson equation. The minus sign in front of this term may also prevent in some cases the proper normalization of the spectroscopic amplitudes. This feature can be understood by considering a possible solution near such a spurious pole. The normalization is determined by the derivative of the self-energy at this energy [13] and will not yield a correct result on account of the additional minus sign when the third diagram dominates. In addition, each of the first two terms in Fig. 1 ignores the Pauli correlations between the freely propagating line and the quasiparticles forming the phonons, as noted in [7]. In the present work a formalism is pursued that sums the contribution of the $\mathrm{pp}$ and ph phonons to the self-energy to all orders avoiding the subtraction of the second-order diagram. The treatment of Pauli correlations is improved over methods that employ ph RPA phonons in the self-energy since all exchange terms at the $2 \mathrm{p} 1 \mathrm{~h}$ level are consistently included.

Other approaches have been proposed in the literature that attempt to extend the nature of the phonon correlations included by performing massive summations of diagrams [1618]. Nevertheless, a consistent resummation of both pp and ph phonons to all orders has not been achieved in these papers. The main problem in pursuing such an infinite summation of diagrams for the $2 \mathrm{p} 1 \mathrm{~h}$ propagator, which includes both pp and ph RPA correlations, is related to the fact that a two-body interaction can invert the sense of propagation of only two lines (i.e., change at most two holes in two particles and vice versa) while the third line continues to propagate in the original direction. In this way, a propagator depending on more than two times is generated. It is therefore necessary first to consider an exact formulation involving the four-time Green's function for the $2 \mathrm{p} 1 \mathrm{~h}$ propagator. Direct application of four-time propagator equations presumably will remain impractical for the forseeable future. Appropriate approximations to this equation are therefore necessary to construct the relevant two-times Green's functions that contain the soughtafter correlations. The scheme studied in this paper consists in computing the RPA phonons in the pp and ph channels, separately, and then summing them to all orders employing a Faddeev technique $[19,20]$.

A nontrivial problem in the implementation of the Fad- deev equations is the appearance of spurious solutions $[21,22]$, which also have to be considered for the $2 \mathrm{p} 1 \mathrm{~h}$ propagator. As pointed out in [23], the spurious eigenstates are easy to recognize for the few-body problem since they also diagonalize the unperturbed Hamiltonian. Their main features are that their eigenvalues are known and that their wave-function amplitudes sum up to zero. The situation is more complicated in the many-body problem when the Faddeev technique is employed. In particular, the fulfillment of closure relations for $\mathrm{pp}$ and $\mathrm{ph}$ amplitudes is related to the behavior of the spurious Faddeev eigenstates. Without a consistent treatment of this relation the spurious solutions will mix with the physically meaningful ones. Applying the Faddeev technique to the many-body problem, it is important to solve for all physical solutions that contribute to the selfenergy. Thus, it is necessary to develop a formalism in which the spurious solutions are correctly separated from the physical ones.

The practical implementation of the present Faddeev scheme is beyond the scope of this paper. The resulting set of equations require a great deal of computational effort, especially when dressed propagators are employed. Nevertheless, it appears that they can be solved in practice and results using this formalism will be presented elsewhere.

In Sec. II we briefly describe the SCGF approach based on the Dyson equation and present the exact Faddeev formalism for the four-time $2 \mathrm{p} 1 \mathrm{~h}$ propagator. The construction of a consistent formulation for the two-time $2 \mathrm{p} 1 \mathrm{~h}$ propagator including the propagation of the pp and ph RPA phonons to all orders is presented in Sec. III. Although so far only the $2 \mathrm{p} 1 \mathrm{~h}$ propagator has been mentioned, it should be understood that the corresponding two-hole-one-particle $(2 \mathrm{~h} 1 \mathrm{p})$ propagator must be included in the calculation of the nucleon selfenergy. In the present work no coupling terms are considered that transform the $2 \mathrm{p} 1 \mathrm{~h}$ into the $2 \mathrm{~h} 1 \mathrm{p}$ propagators (or vice versa). For this reason the same technique can be used for both propagators and we will use the generic $2 \mathrm{p} 1 \mathrm{~h}$ to represent both. In Sec. IV the appearance and treatment of spurious solutions is discussed. Some technical details are relegated to the Appendices. Conclusions are drawn in Sec. V.

\section{SELF-CONSISTENT GREEN'S FUNCTION APPROACH AND 2p1h PROPAGATOR}

\section{A. Self-energy and $2 \mathrm{p} 1 \mathrm{~h}$ propagator}

We consider a finite system of $A$ fermions interacting by means of a two-body interaction $\hat{V}$. As usual, one may introduce an appropriate mean-field potential $\hat{U}$ to localize the nucleons and split the Hamiltonian into an unperturbed onebody part $\hat{H}_{0}=\hat{T}+\hat{U}$ and a residual interaction $\hat{H}_{1}=\hat{V}-\hat{U}$. 
Since we are mainly interested in low-lying bound states of finite systems, we consider s.p. states with discrete quantum numbers. As a basis, we choose the set of s.p. states $\{\alpha\}$ that diagonalize $\hat{H}_{0}$ with corresponding eigenvalues $\varepsilon_{\alpha}^{0}$. The total Hamiltonian can then be written as

$$
\begin{aligned}
\hat{H}= & \hat{H}_{0}+\hat{H}_{1} \\
= & \sum_{\alpha} \varepsilon_{\alpha}^{0} c_{\alpha}^{\dagger} c_{\alpha} \\
& +\left(\frac{1}{4} \sum_{\alpha \beta \gamma \delta} V_{\alpha \beta, \gamma \delta} c_{\alpha}^{\dagger} c_{\beta}^{\dagger} c_{\delta} c_{\gamma}-\sum_{\alpha \beta} U_{\alpha, \beta} c_{\alpha}^{\dagger} c_{\beta}\right),
\end{aligned}
$$

where $c_{\alpha}^{\dagger}\left(c_{\alpha}\right)$ are the creation (destruction) operators of a particle in the state $\alpha, V_{\alpha \beta, \gamma \delta}$ are the antisymmetrized matrix elements of $V$, and $U_{\alpha, \beta}$ correspond to the matrix elements of $U$.

The one-body propagator of the $A$-body system with ground state $\left|\Psi_{0}^{A}\right\rangle$ is defined as $[24,25]$

$$
g_{\alpha \beta}(\tau)=-i\left\langle\Psi_{0}^{A}\left|T\left[c_{\alpha}(\tau) c_{\beta}^{\dagger}(0)\right]\right| \Psi_{0}^{A}\right\rangle,
$$

where $c_{\alpha}^{\dagger}(t)$ and $c_{\alpha}(t)$ now correspond to operators in the Heisenberg picture. In the Lehmann representation, all the eigenvalues of the excited states of the systems with $A+1$ and $A-1$ particles appear, as well as their spectroscopic amplitudes for transitions to those states that are relevant for comparison with experimental data.
The propagator $g_{\alpha \beta}(\tau)$ can be obtained as the sum of an infinite set of diagrams, built from interaction vertices and unperturbed s.p. propagators $g_{\alpha \beta}^{(0)}$ corresponding to $\hat{H}_{0}$. In the nuclear case, a strong coupling exists between the s.p. degree of freedom and both collective low-lying states as well as high-lying states. The latter coupling is related to the strong short-range repulsion in the nuclear force. The resulting fragmentation of the s.p. strength, as observed in experimental data, suggests that this feature must already be included in the description of these couplings. For this reason, selfconsistent one-body propagators need to be considered in the construction of the nucleon self-energy. This selfconsistency feature also emerges in an exact formulation, involving the coupling to two-, three-, and A-body propagators, which can be derived using the equation of motion method [26]. In short, this means that for the nuclear case one needs to develop the perturbation theory in terms of the dressed propagator (2) approximated in an appropriate way.

The approach we use here consists in computing $g_{\alpha \beta}(\tau)$ as a solution of the Dyson equation

$$
g_{\alpha \beta}(\tau)=g_{\alpha \beta}^{(0)}(\tau)+g_{\alpha \gamma}^{(0)}\left(\tau-t_{1}\right) \sum_{\gamma \delta}^{*}\left(t_{1}-t_{2}\right) g_{\delta \beta}\left(t_{2}\right),
$$

where $\Sigma_{\alpha \beta}^{*}(\tau)$ is the irreducible self-energy. Here and in the following, we employ the convention of summing over all repeated indices and integrate from $-\infty$ to $+\infty$ over all repeated time variables, unless specified otherwise.

By considering the equation of motion for $g_{\alpha \beta}(\tau)$, one obtains that $\Sigma_{\alpha \beta}^{*}(\tau)$ can be written as the sum of two terms

$$
\Sigma_{\alpha \beta}^{*}(\tau)=\Sigma_{\alpha \beta}^{H F}+V_{\alpha \lambda, \mu \nu} R_{\mu \nu \lambda, \gamma \delta \varepsilon}\left(\tau^{-}, \tau, \tau^{+} ; 0^{+}, 0,0^{-}\right) V_{\gamma \delta, \beta \varepsilon},
$$

where $\Sigma_{\alpha \beta}^{H F}$ represents the (time independent) Hartree-Fock part of the self-energy, which can be computed from the solution $g_{\alpha \beta}(\tau)$ itself. The 2 p1h propagator $R$, appearing in the last term of Eq. (4), contains the sum of all so-called one-particle irreducible diagrams that cannot be separated by cutting a single line. These terms are included in the $2 \mathrm{p} 1 \mathrm{~h}$ Green's function $g_{\mu \nu \lambda, \alpha \beta \gamma}^{2 p 1 h}$ defined below. The relation between $R$ and $g^{2 p 1 h}$ is given by [27]

$$
R_{\mu \nu \lambda, \alpha \beta \gamma}\left(t_{1}, t_{2}, t_{3} ; t_{4}, t_{5}, t_{6}\right)=g_{\mu \nu \lambda, \alpha \beta \gamma}^{2 p 1 h}\left(t_{1}, t_{2}, t_{3} ; t_{4}, t_{5}, t_{6}\right)-g_{\mu \nu, \lambda \eta}^{I I}\left(t_{1}, t_{2} ; t_{3}, t^{\prime}\right) g_{\eta \sigma}^{-1}\left(t^{\prime}-t^{\prime \prime}\right) g_{\gamma \sigma, \alpha \beta}^{I I}\left(t_{6}, t^{\prime \prime} ; t_{4}, t_{5}\right),
$$

in which $g_{\eta \sigma}^{-1}$ is the inverse of the one-body Green's function (2) and $g^{I I}$ and $g^{2 p 1 h}$ are the four- and six-point Green's functions defined as

$$
\begin{aligned}
& g_{\alpha \beta, \gamma \delta}^{I I}\left(t_{1}, t_{2} ; t_{3}, t_{4}\right) \\
& \quad=-i\left\langle\Psi_{0}^{A}\left|T\left[c_{\beta}\left(t_{2}\right) c_{\alpha}\left(t_{1}\right) c_{\gamma}^{\dagger}\left(t_{3}\right) c_{\delta}^{\dagger}\left(t_{4}\right)\right]\right| \Psi_{0}^{A}\right\rangle
\end{aligned}
$$

$$
\begin{aligned}
g_{\mu \nu \lambda, \alpha \beta \gamma}^{2 p 1 h}\left(t_{1}, t_{2}, t_{3} ; t_{4}, t_{5}, t_{6}\right) & \\
= & -i\left\langle\Psi_{0}^{A}\right| T\left[c_{\lambda}^{\dagger}\left(t_{3}\right) c_{\nu}\left(t_{2}\right) c_{\mu}\left(t_{1}\right) c_{\alpha}^{\dagger}\left(t_{4}\right) c_{\beta}^{\dagger}\left(t_{5}\right) c_{\gamma}\left(t_{6}\right)\right] \\
& \times\left|\Psi_{0}^{A}\right\rangle,
\end{aligned}
$$

respectively.

The propagator $R_{\mu \nu \lambda, \alpha \beta \gamma}$ is the solution of the following equation that has a similar form as the Bethe-Salpeter equation for $\mathrm{pp}$ and $\mathrm{ph}$ propagators:

and 


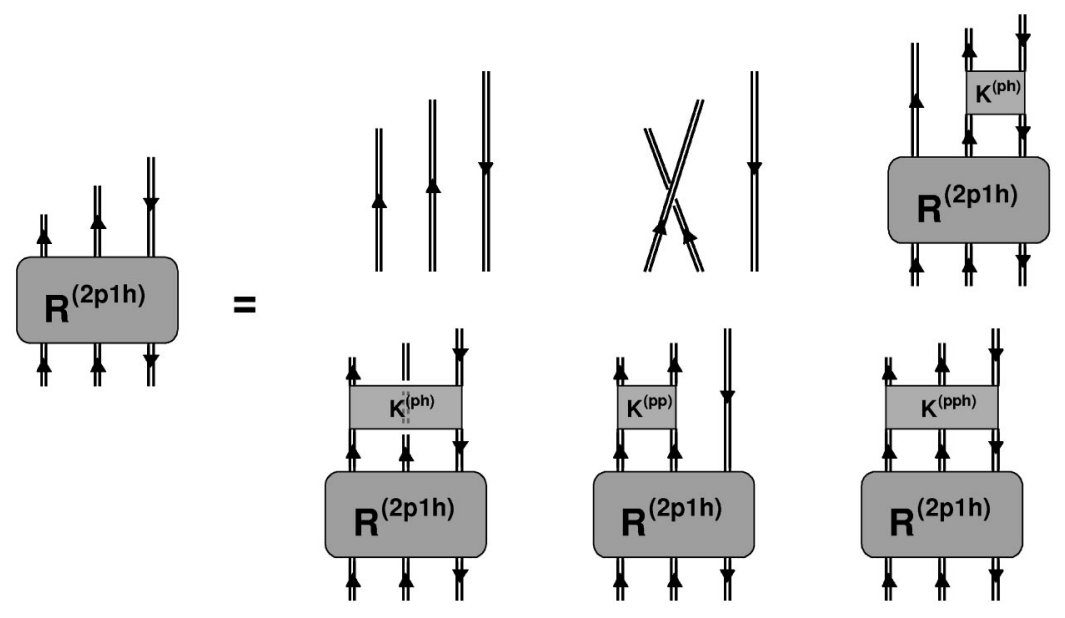

FIG. 2. Bethe-Salpeter equation (8) for the $2 \mathrm{p} 1 \mathrm{~h}$ propagator with vertices given by Eq. (9). The irreducible interaction vertices for the $\mathrm{pp}$ and two ph channels are denoted by $K^{p p}$ and $K^{p h}$, respectively. The irreducible vertex involving all three lines simultaneously is denoted by $K^{p p h}$.

$$
\begin{aligned}
R_{\mu \nu \lambda, \alpha \beta \gamma}\left(t_{1}, t_{2}, t_{3} ; t_{4}, t_{5}, t_{6}\right) \\
=g_{\mu \alpha}\left(t_{1}-t_{4}\right) g_{\nu \beta}\left(t_{2}-t_{5}\right) g_{\gamma \lambda}\left(t_{6}-t_{3}\right) \\
\quad-g_{\nu \alpha}\left(t_{2}-t_{4}\right) g_{\mu \beta}\left(t_{1}-t_{5}\right) g_{\gamma \lambda}\left(t_{6}-t_{3}\right) \\
\quad+g_{\mu \mu^{\prime}}\left(t_{1}-t_{1}^{\prime}\right) g_{\nu \nu^{\prime}}\left(t_{2}-t_{2}^{\prime}\right) g_{\lambda^{\prime} \lambda}\left(t_{3}^{\prime}-t_{3}\right) \\
\quad \times K_{\mu^{\prime} \nu^{\prime} \lambda^{\prime}, \alpha^{\prime} \beta^{\prime} \gamma^{\prime}}\left(t_{1}^{\prime}, t_{2}^{\prime}, t_{3}^{\prime} ; t_{4}^{\prime}, t_{5}^{\prime}, t_{6}^{\prime}\right) \\
\quad \times R_{\alpha^{\prime} \beta^{\prime} \gamma^{\prime}, \alpha \beta \gamma}\left(t_{4}^{\prime}, t_{5}^{\prime}, t_{6}^{\prime} ; t_{4}, t_{5}, t_{6}\right),
\end{aligned}
$$

which is shown in Fig. 2 in terms of Feynman diagrams. The interaction vertex, also shown in Fig. 2, is given [27] by

$$
\begin{aligned}
K_{\mu \nu \lambda, \alpha \beta \gamma}\left(t_{1}, t_{2}, t_{3} ; t_{4}, t_{5}, t_{6}\right) \\
=K_{\nu \lambda, \beta \gamma}^{(p h)}\left(t_{2}, t_{3} ; t_{5}, t_{6}\right) g_{\mu \alpha}^{-1}\left(t_{1}-t_{4}\right) \\
\quad+K_{\mu \lambda, \alpha \gamma}^{(p h)}\left(t_{1}, t_{3} ; t_{4}, t_{6}\right) g_{\nu \beta}^{-1}\left(t_{2}-t_{5}\right) \\
\quad+K_{\mu \nu, \alpha \beta}^{(p p)}\left(t_{1}, t_{2} ; t_{4}, t_{5}\right) g_{\gamma \lambda}^{-1}\left(t_{6}-t_{3}\right) \\
\quad+K_{\mu \nu \lambda, \alpha \beta \gamma}^{(p p h)}\left(t_{1}, t_{2}, t_{3} ; t_{4}, t_{5}, t_{6}\right) .
\end{aligned}
$$

In Eq. (9), $K^{(p p)}$ and $K^{(p h)}$ represent the pp and ph irreducible vertices while $K^{(p p h)}$ is the 2 p1h irreducible vertex. It should be noted that in Eq. (4) the propagator $R_{\mu \nu \lambda, \alpha \beta \gamma}$ is only required at two times and therefore its complete knowledge, as given by Eq. (5), is not necessary to solve the Dyson equation. On the other hand, the dependence on the time variables $t_{1}, t_{2}$, and $t_{3}$ is employed in the Bethe-Salpeter equation (8), thus requiring that at least a four-time object be employed to solve for the $2 \mathrm{p} 1 \mathrm{~h}$ motion exactly.

Equations (3), (4), and (8) together form a set of coupled equations, where the same propagator, which solves the Dyson equation (3), appears as input in the Bethe-Salpeter equation (8). If the irreducible vertices $K^{(p p)}, K^{(p h)}$, and $K^{(p p h)}$ are also expressed in terms of the $g_{\alpha \beta}(\tau)$, then Eqs. (3) and (8) will generate a self-consistent expansion. Obviously, Eq. (8) and the irreducible vertex (9) represent the exact solution for $R$ and therefore require a suitable approximation.

\section{B. Faddeev-Bethe-Salpeter equations}

Equation (8) can be reduced to a set of coupled equations in a way similar to the method proposed by Faddeev to solve the three-body problem $[19,28]$. The inclusion of $\mathrm{pp}$ and $\mathrm{ph}$ RPA phonons in a consistent way requires this Faddeev approach since it provides a natural framework for correctly iterating quantities that have already been summed to all orders like these RPA phonons. In the present work we will neglect the contribution of the irreducible $K^{(p p h)}$ term in Eq. (9) since it leads to the coupling of higher order particle-hole terms than already considered in the following. We will therefore require only three Faddeev components. Following standard notation in the literature [20], $R_{\mu \nu \lambda, \alpha \beta \gamma}^{(i)}$ will represent the component related to all diagrams ending with a vertex between legs $j$ and $k$ with $(i, j, k)$ cyclic permutations of $(1,2,3)$. We will employ the convention in which the third leg propagates in the opposite direction with respect to the first two. The Faddeev components $R^{(i)}$ can be written in terms of the $2 \mathrm{p} 1 \mathrm{~h}$ propagator $R$ and the contribution of the three dressed but noninteracting s.p. propagators. This definition is given in detail here for all three components, omitting explicit reference to the time variables for convenience of notation

$$
\begin{aligned}
R_{\mu \nu \lambda, \alpha \beta \gamma}^{(1)}= & g_{\nu \epsilon} g_{\rho \lambda} K_{\epsilon \rho, \eta \sigma}^{(p h)} R_{\mu \eta \sigma, \alpha \beta \gamma} \\
& +\frac{1}{2}\left(g_{\mu \alpha} g_{\nu \beta} g_{\gamma \lambda}-g_{\nu \alpha} g_{\mu \beta} g_{\gamma \lambda}\right), \\
R_{\mu \nu \lambda, \alpha \beta \gamma}^{(2)}= & g_{\mu \epsilon} g_{\rho \lambda} K_{\epsilon \rho, \eta \sigma}^{(p h)} R_{\eta \nu \sigma, \alpha \beta \gamma} \\
& +\frac{1}{2}\left(g_{\mu \alpha} g_{\nu \beta} g_{\gamma \lambda}-g_{\nu \alpha} g_{\mu \beta} g_{\gamma \lambda}\right), \\
R_{\mu \nu \lambda, \alpha \beta \gamma}^{(3)}= & g_{\mu \epsilon} g_{\nu \rho} K_{\epsilon \rho, \eta \sigma}^{(p p)} R_{\eta \sigma \lambda, \alpha \beta \gamma} \\
& +\frac{1}{2}\left(g_{\mu \alpha} g_{\nu \beta} g_{\gamma \lambda}-g_{\nu \alpha} g_{\mu \beta} g_{\gamma \lambda}\right) .
\end{aligned}
$$

The factor $\frac{1}{2}$ in Eqs. (10) properly takes into account the exchange symmetry between the parallel lines in the Faddeev equations. With these definitions the full propagator (5) 
is given by

$$
R_{\mu \nu \lambda, \alpha \beta \gamma}=\sum_{i=1,2,3} R_{\mu \nu \lambda, \alpha \beta \gamma}^{(i)}-\frac{1}{2}\left(g_{\mu \alpha} g_{\nu \beta} g_{\gamma \lambda}-g_{\nu \alpha} g_{\mu \beta} g_{\gamma \lambda}\right) .
$$

The Faddeev equations now take the following form:

$$
\begin{aligned}
R_{\mu \nu \lambda, \alpha \beta \gamma}^{(i)}= & \frac{1}{2}\left(g_{\mu \alpha} g_{\nu \beta} g_{\gamma \lambda}-g_{\nu \alpha} g_{\mu \beta} g_{\gamma \lambda}\right) \\
& +g_{\mu \mu^{\prime}} g_{\nu \nu^{\prime}} g_{\lambda^{\prime} \lambda} \Gamma_{\mu^{\prime} \nu^{\prime} \lambda^{\prime}, \mu^{\prime \prime} \nu^{\prime \prime} \lambda^{\prime \prime}}^{(i)}\left(R_{\mu^{\prime \prime} \nu^{\prime \prime} \lambda^{\prime \prime}, \alpha \beta \gamma}^{(j)}\right. \\
& \left.+R_{\mu^{\prime \prime} \nu^{\prime \prime} \lambda^{\prime \prime}, \alpha \beta \gamma}^{(k)}\right), \quad i=1,2,3
\end{aligned}
$$

where the $\Gamma_{\mu \nu \lambda, \alpha \beta \gamma}^{(i)}$ vertices obey the following symmetry relations and are defined by

$$
\begin{aligned}
& \Gamma_{\mu \nu \lambda, \alpha \beta \gamma}^{(1)}\left(t_{1}, t_{2}, t_{3} ; t_{4}, t_{5}, t_{6}\right) \\
& \quad=\Gamma_{\nu \mu \lambda, \beta \alpha \gamma}^{(2)}\left(t_{2}, t_{1}, t_{3} ; t_{5}, t_{4}, t_{6}\right) \\
& \quad=g_{\mu \alpha}^{-1}\left(t_{1}-t_{4}\right) \widetilde{\Gamma}_{\nu \lambda, \beta \gamma}^{(p h)}\left(t_{2}, t_{3} ; t_{5}, t_{6}\right), \\
& \Gamma_{\mu \nu \lambda, \alpha \beta \gamma}^{(3)}\left(t_{1}, t_{2}, t_{3} ; t_{4}, t_{5}, t_{6}\right) \\
& \quad=\Gamma_{\nu \mu \lambda, \beta \alpha \gamma}^{(3)}\left(t_{2}, t_{1}, t_{3} ; t_{5}, t_{4}, t_{6}\right) \\
& \quad=g_{\gamma \lambda}^{-1}\left(t_{6}-t_{3}\right) \widetilde{\Gamma}_{\mu \nu, \alpha \beta}^{(p p)}\left(t_{1}, t_{2} ; t_{4}, t_{5}\right) .
\end{aligned}
$$

The gamma matrices $\tilde{\Gamma}^{(p p)}$ and $\widetilde{\Gamma}^{(p h)}$ are the four-point functions that solve the Bethe-Salpeter equation for the $\mathrm{pp}$ and $\mathrm{ph}$ motion. These vertex functions contain the $\mathrm{pp}$ and $\mathrm{ph}$ phonons and can be written as

$$
\begin{aligned}
\tilde{\Gamma}_{\gamma \delta, \alpha \beta}^{(p p)}\left(t_{1}, t_{2} ; t_{3}, t_{4}\right) & \\
= & K_{\gamma \delta, \alpha \beta}^{(p p)}\left(t_{1}, t_{2} ; t_{3}, t_{4}\right)+\widetilde{\Gamma}_{\gamma \delta, \mu \nu}^{(p p)}\left(t_{1}, t_{2} ; t_{1}^{\prime}, t_{2}^{\prime}\right) \\
& \quad \times g_{\mu \eta}\left(t_{1}^{\prime}-t_{3}^{\prime}\right) g_{\nu \sigma}\left(t_{2}^{\prime}-t_{4}^{\prime}\right) K_{\eta \sigma, \alpha \beta}^{(p p)}\left(t_{3}^{\prime}, t_{4}^{\prime} ; t_{3}, t_{4}\right), \\
\tilde{\Gamma}_{\gamma \delta, \alpha \beta}^{(p h)}\left(t_{1}, t_{2} ; t_{3}, t_{4}\right) & \\
= & K_{\gamma \delta, \alpha \beta}^{(p h)}\left(t_{1}, t_{2} ; t_{3}, t_{4}\right)+\widetilde{\Gamma}_{\gamma \delta, \mu \nu}^{(p h)}\left(t_{1}, t_{2} ; t_{1}^{\prime}, t_{2}^{\prime}\right) \\
& \times g_{\mu \eta}\left(t_{1}^{\prime}-t_{3}^{\prime}\right) g_{\sigma \nu}\left(t_{4}^{\prime}-t_{2}^{\prime}\right) K_{\eta \sigma, \alpha \beta}^{(p h)}\left(t_{3}^{\prime}, t_{4}^{\prime} ; t_{3}, t_{4}\right) .
\end{aligned}
$$

\section{APPROXIMATE FADDEEV EQUATIONS FOR 2p1h MOTION}

Apart from neglecting the $K^{(2 p 1 h)}$ vertex, Eq. (12) is otherwise a complete equation for the $2 \mathrm{p} 1 \mathrm{~h}$ propagator. This general equation involves quantities that depend on several times and is therefore too complex to be solved numerically. In order to construct a manageable approximation scheme that includes the relevant physical ingredients, two simplifications will be considered in this section. The first one involves the restriction to two-time $\mathrm{pp}$ and ph vertices that include the respective RPA contributions in these channels. This approximation is the minimum step that maintains the
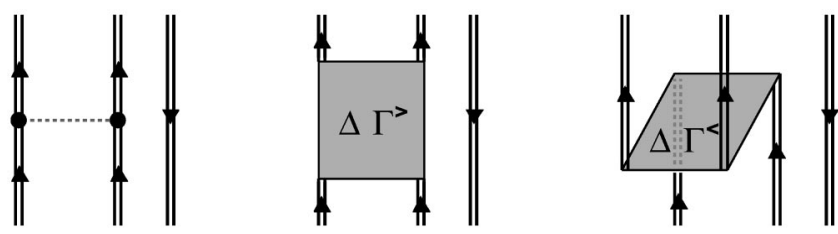

FIG. 3. Diagrams that are included in the definition of the vertex for the pp channel. Here $\Delta \Gamma^{>}$and $\Delta \Gamma^{<}$are the forward- and backward-going part of the energy dependent contribution to the pp DRPA vertex (A1). The contribution of these three diagrams can be factorized in an expression of the form $G^{0>} \Gamma^{(3)} G^{0>}$ only after having redefined the propagators $G^{0>}$ and $\Gamma^{(3)}$ to depend also on the particle and hole fragmentation indices $\left(n, n^{\prime}, k\right)$. The last diagram has a smaller effect on the physical solutions of the problem, although it is essential for the elimination of spurious solutions.

simultaneous inclusion of both $\mathrm{pp}$ and ph collective lowlying excitations in describing the s.p. propagator. Second, it is necessary to simplify Eq. (12) to include only two-times Green's functions. This procedure no longer allows the inversion of the propagation direction of all three lines together. As a result, the Faddeev equations split up in two separate expansions for the $2 \mathrm{p} 1 \mathrm{~h}$ and the $2 \mathrm{~h} 1 \mathrm{p}$ components. Although the hole spectral function is of primary interest for comparison with experimental data, it must be stressed that both $2 \mathrm{p} 1 \mathrm{~h}$ and $2 \mathrm{~h} 1 \mathrm{p}$ components are needed to generate the self-consistent solution for the s.p. propagator. Since the formalism involved is the same for both components, we will describe only the forward-going (2p1h) expansion. The equations for the $2 \mathrm{~h} 1 \mathrm{p}$ case are completely analogous.

\section{A. Faddeev equations}

To construct the present approximation scheme, it is more convenient to use the energy representation. The corresponding Lehmann representation of the s.p. propagator (2) is given by

$$
g_{\alpha \beta}(\omega)=\sum_{n} \frac{\left(\mathcal{X}_{\alpha}^{n}\right)^{*} \mathcal{X}_{\beta}^{n}}{\omega-\varepsilon_{n}^{+}+i \eta}+\sum_{k} \frac{\mathcal{Y}_{\alpha}^{k}\left(\mathcal{Y}_{\beta}^{k}\right)^{*}}{\omega-\varepsilon_{k}^{-}-i \eta}
$$

where $\mathcal{X}_{\alpha}^{n}=\left\langle\Psi_{n}^{A+1}\left|c_{\alpha}^{\dagger}\right| \Psi_{0}^{A}\right\rangle\left(\mathcal{Y}_{\alpha}^{k}=\left\langle\Psi_{k}^{A-1}\left|c_{\alpha}\right| \Psi_{0}^{A}\right\rangle\right)$ are the spectroscopic amplitudes for the excited states of a system with $A+1(A-1)$ particles and the poles $\varepsilon_{n}^{+}=E_{n}^{A+1}-E_{0}^{A}$ $\left(\varepsilon_{k}^{-}=E_{0}^{A}-E_{k}^{A-1}\right)$ correspond to the excitation energies with respect to the $A$-body ground state. In Eq. (15) and in the following, we use the indices $n$ and $k$ to enumerate the fragments associated with the one-particle and one-hole excitations, respectively.

Employing the bare interaction $V_{\alpha \beta, \gamma \delta}$ for the vertices $K^{(p p)}$ and $K^{(p h)}$, the Bethe-Salpeter equations (14) reduce to the usual dressed RPA (DRPA) equations [29,30]. The solutions of these equations depend only on two times. These pp and ph phonons correspond to the dressed version of the phonons that are considered in Ref. [7] (see also Fig. 1). These excitations describe the correlations that we aim to iterate to all orders and, subsequently, to include in the selfenergy as explained in the introduction. These DRPA solutions can then be substituted in Eqs. (13) to generate the $\Gamma^{(i)}$ 

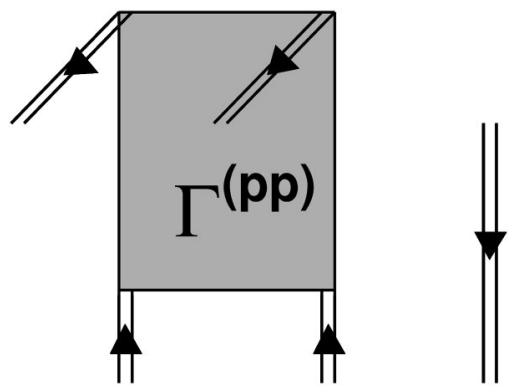

FIG. 4. A combination of two diagrams of the type shown here can be used to connect the $2 \mathrm{p} 1 \mathrm{~h}$ and $2 \mathrm{~h} 1 \mathrm{p}$ propagators. Diagrams like these are not included in the present approximation scheme. Nevertheless, their contribution appears in the normalization of spectroscopic amplitudes. Explicit time-ordering is implied in this diagram.

matrices to be used in the Faddeev expansion. Both the forward- and backward-going components of the DRPA solutions are included into the expansion as illustrated in Fig. 3. This is crucial in order to eliminate the spurious solutions of the Faddeev equations as will be explained in Sec. IV and Appendix C.

The working expression for the $\Gamma^{(i)}$ matrices, which depends on only two times (or equivalently one energy), is given in some detail in Appendix A. Here we only need to stress that the resulting $\Gamma^{(i)}$ 's cannot invert the freely propagating line from hole to particle or vice versa, i.e., they cannot connect the $2 \mathrm{p} 1 \mathrm{~h}$ amplitudes with the $2 \mathrm{~h} 1 \mathrm{p}$ ones. For this reason, the pp and ph phonons will be summed only in one time direction in a TDA way contributing separately to the $2 \mathrm{p} 1 \mathrm{~h}$ and $2 \mathrm{~h} 1 \mathrm{p}$ propagators. The reader may notice that two contributions of the type shown in Fig. 4 can connect the $2 \mathrm{p} 1 \mathrm{~h}$ and the $2 \mathrm{~h} 1 \mathrm{p}$ propagators. The inclusion of such terms leads to the simultaneous propagation of two phonons that requires an extension of the approximation presented in this paper. Since these terms are expected to contribute only in higher order, we will neglect them in the following. We note that the collective RPA correlations in the pp and ph channels have already been computed through Eqs. (14) and therefore remain properly included in our approximation.

The remaining complication, related to the use of dressed propagators, concerns the interactions vertices (13). As explained in Appendix $\mathrm{A}$, the $\Gamma^{(i)}$ and the propagators $R^{(i)}$ need to be redefined in such a way that their matrix elements also depend on the indices $\left(n, n^{\prime}, k\right)$, which label the fragments of the propagators. This implies that the eigenvalue equations will involve summations on both the s.p. indices $(\alpha, \beta, \gamma)$ and the ones corresponding to the fragmentation, $\left(n_{\alpha}, n_{\beta}, k_{\gamma}\right)$. The $2 \mathrm{p} 1 \mathrm{~h}$ propagator and its Faddeev components, as defined in Eqs. (5) and (10), are recovered only at the end by summing the solutions over all values of $\left(n_{\alpha}, n_{\beta}, k_{\gamma}\right)$ and $\left(n_{\mu}, n_{\nu}, k_{\lambda}\right)$.

Putting together all the above considerations, the resulting approximation to the Faddeev equations (12) can be rewritten in a way where all the propagators involved depend only on one energy variable (or two time variables). The forwardgoing part of this expansion can be written as follows:

$$
\begin{aligned}
R_{\mu n_{\mu} \nu n_{\nu} \lambda k_{\lambda}, \alpha n_{\alpha} \beta n_{\beta} \gamma k_{\gamma}}^{(i)}(\omega) \\
=\frac{1}{2}\left[G_{\mu n_{\mu} \nu n_{\nu} \lambda k_{\lambda}, \alpha n_{\alpha} \beta n_{\beta} \gamma k_{\gamma}}^{0>}(\omega)\right. \\
\left.\quad-G_{\nu n_{\nu} \mu n_{\mu} \lambda k_{\lambda}, \alpha n_{\alpha} \beta n_{\beta} \gamma k_{\gamma}}^{0>}(\omega)\right] \\
\quad+G_{\nu n_{\nu} \mu n_{\mu} \lambda k_{\lambda}, \mu^{\prime} n_{\mu}^{\prime} \nu^{\prime} n_{\nu}^{\prime} \lambda^{\prime} k_{\lambda}^{\prime}}^{0>}(\omega) \\
\quad \times \Gamma_{\nu^{\prime} n_{\nu}^{\prime} \mu^{\prime} n_{\mu}^{\prime}{ }^{\prime} k_{\lambda}^{\prime}, \mu^{\prime \prime} n_{\mu}^{\prime \prime} \nu^{\prime \prime} n_{\nu}^{\prime \prime} \lambda^{\prime \prime} k_{\lambda}^{\prime \prime}}^{(i)}(\omega) \\
\quad \times\left[R_{\mu^{\prime \prime} n_{\mu}^{\prime \prime} \nu^{\prime \prime} n_{\nu}^{\prime \prime} \lambda^{\prime \prime} k_{\lambda}^{\prime \prime}, \alpha n_{\alpha} \beta n_{\beta} \gamma k_{\gamma}}^{(j)}(\omega)\right. \\
\left.\quad+R_{\mu^{\prime \prime} n_{\mu}^{\prime \prime} \nu^{\prime \prime} n_{\nu}^{\prime \prime} \lambda^{\prime \prime} k_{\lambda}^{\prime \prime}, \alpha n_{\alpha} \beta n_{\beta} \gamma k_{\gamma}}(\omega)\right], \quad i=1,2,3,
\end{aligned}
$$

where $G^{0>}$ is the forward-going part of the 2 p1h propagator for three dressed but noninteracting lines. Using the notations introduced after Eq. (15) we have

$$
\begin{aligned}
G_{\mu n_{\mu} \nu n_{\nu} \lambda k_{\lambda}, \alpha n_{\alpha} \beta n_{\beta} \gamma k_{\gamma}}^{0>}(\omega)= & \delta_{n_{\mu}, n_{\alpha}} \delta_{n_{\nu}, n_{\beta}} \delta_{k_{\lambda}, k_{\gamma}} \\
& \times \frac{\left(\mathcal{X}_{\mu}^{n_{\mu}} \mathcal{X}_{\nu}^{n_{\nu}} \mathcal{Y}_{\lambda}^{k_{\lambda}}\right) * \mathcal{X}_{\alpha}^{n_{\alpha}} \mathcal{X}_{\beta}^{n_{\beta}} \mathcal{Y}_{\gamma}^{k_{\gamma}}}{\omega-\left(\varepsilon_{n_{\alpha}}^{+}+\varepsilon_{n_{\beta}}^{+}-\varepsilon_{k \gamma}^{-}\right)+i \eta} .
\end{aligned}
$$

Equation (16), together with the $\Gamma^{(i)}$ 's given in Appendix A, approximate the general "Faddeev-Bethe-Salpeter" expansion to a tractable set of equations involving only twotime objects. It is important to note that these equations are still expressed in terms of the self-consistent solution $g_{\alpha \beta}(\omega)$ and include both pp and ph RPA phonons in a correct way. Thus they maintain all the features relevant for the physics we aim to describe.

\section{B. Faddeev amplitudes}

Equation (16) involves the use of propagators depending on a large number of indices. As a consequence, the dimension of the problem could easily grow up to a point where no practical application is feasible for a real system. This difficulty can be overcome by introducing a new set of spectroscopic amplitudes that depend only on the indices labeling the particle and hole fragments $\left(n, n^{\prime}, k\right)[31,32]$. Thereby the problem is reexpressed by changing from the basis of s.p. states $\{\alpha\}$, used in definitions (5) and (10), to a new formulation constructed in terms of the fragments labeled by $\{n, k\}$. This procedure also allows to rewrite the eigenvalue and normalization conditions corresponding to Eq. (16) in a more concise way. As long as the interaction elements $V_{\alpha \beta, \gamma \delta}$ are energy independent, all the solutions can then be obtained through a single diagonalization. This approach is particularly satisfactory from a physical point of view since the equations reflect the mixing of the $2 \mathrm{p} 1 \mathrm{~h}$ states represented by the $\left(n, n^{\prime}, k\right)$ fragments. This new formulation does not introduce any further approximation. Nevertheless, since it appears relevant for a practical solution of the problem, we will describe it in the following. 
The Lehmann representation of the Faddeev components $R_{\mu n_{\mu} \nu n_{\nu} \lambda k_{\lambda}, \alpha n_{\alpha} \beta n_{\beta} \gamma k_{\gamma}}^{(i)}$ contains all the poles $\varepsilon_{m}^{F d}$ of the $2 \mathrm{p} 1 \mathrm{~h}$ propagator, each with its own residue. One obtains for these components

$$
\begin{aligned}
R_{\mu n_{\mu} \nu n_{\nu} \lambda k_{\lambda}, \alpha n_{\alpha} \beta n_{\beta} \gamma k_{\gamma}}^{(i)}= & \sum_{m} \frac{\left(\beta_{\mu n_{\mu} \nu n \nu_{\nu} \lambda k_{\lambda}}^{(i)}\right)^{*} b_{\alpha n_{\alpha} \beta n_{\beta} \gamma k_{\gamma}}^{m}}{\omega-\varepsilon_{m}^{F d}+i \eta} \\
& +R_{\text {free }}^{(i)}(\omega),
\end{aligned}
$$

where the superscript $m$ labels the solutions of Faddeev equations. In Eq. (18), $R_{\text {free }}^{(i)}$ represent components containing the same poles as $G^{0>}(17)$. The sum of these terms cancels exactly the contribution of the three freely propagating lines in Eq. (11), leaving in the Lehmann representation of the Faddeev propagator only those poles $\varepsilon_{m}^{F d}$, that correspond to correlated $2 \mathrm{p} 1 \mathrm{~h}$ states. This is most easily demonstrated by applying the DRPA equations to both sides of Eq. (16).

The vectors $\beta_{\mu_{n}{ }_{\mu} \nu n_{\nu} \lambda k_{\lambda}}^{(i), m}$ represent the amplitudes of the three Faddeev components and sum up to the residues of the full propagator

$$
b_{\alpha n_{\alpha} \beta n_{\beta} \gamma k_{\gamma}}^{m}=\sum_{i=1,2,3} \beta_{\alpha n_{\alpha} \beta n_{\beta} \gamma k_{\gamma}}^{(i), m} .
$$

We now define new Faddeev amplitudes $x_{n_{1} n_{2}{ }^{k}}^{(i), m}$ that are related to the $\beta^{(i)}$ 's in such a way that [31]

$$
\beta_{\alpha n_{1} \beta n_{2} \gamma k}^{(i), m}=\mathcal{X}_{\alpha}^{n_{1}} \mathcal{X}_{\beta}^{n_{2}} \mathcal{Y}_{\gamma}^{k} x_{n_{1} n_{2} k}^{(i), m},
$$

where no summation is performed over the particle and hole indices $n_{1}, n_{2}$, and $k$. We also introduce the notation for the spectroscopic amplitude, analogous to Eq. (19)

$$
x_{n_{1} n_{2} k}^{m}=\sum_{i=1,2,3} x_{n_{1} n_{2} k}^{(i), m} .
$$

In general, $x_{n_{1} n_{2} k}$ and the components $x_{n_{1} n_{2} k}^{(i)}$ define fourvectors $\mathbf{x}$ and $\mathbf{x}^{(i)}$ all belonging to the same linear space. It is useful to split up the latter in two spaces $V_{A}$ and $V_{S}$ containing all the vectors that are antisymmetric and symmetric with respect to the exchange of the two particle indices $n_{1}$ and $n_{2}$, respectively. Thus,

$$
\mathbf{x}, \mathbf{x}^{(i)} \in V_{A} \otimes V_{S} .
$$

We also define a vector $\mathbf{X}$ containing all the three components

$$
\mathbf{X}=\left(\begin{array}{c}
\mathbf{x}^{(1)} \\
\mathbf{x}^{(2)} \\
\mathbf{x}^{(3)}
\end{array}\right) \in V_{A}^{3} \otimes V_{S}^{3} .
$$

Here and in the following, we use the convention to denote vectors with lower case boldface and operators (matrices) with plain capital letters belonging to the space $V_{A} \otimes V_{S}$. Both vectors and matrices in the space $V_{A}^{3} \otimes V_{S}^{3}$ are denoted by capital boldface letters. We will also use $I$ for the identity matrix in the $V_{A} \otimes V_{S}$ space and the superscript $e x$ to indicate the vectors obtained by exchanging the two particle indices [thus, $I^{e x}$ is the operator exchanging $n_{1}$ and $n_{2}$ in Eqs. (20) and (21)].

\section{Faddeev Hamiltonian}

The eigenvalue equation for the Faddeev expansion can be obtained by substituting the Lehmann representation (18) into Eq. (16) and extracting the residues $\varepsilon_{m}^{F d}$ of the poles. After some algebra, one obtains the following set of equations in terms of the $\mathbf{x}^{(i)}$ vectors

$\mathbf{x}^{(i)}=\left[H^{(i)} H^{(i)^{\dagger}}+U^{(i)} \frac{1}{\omega-D^{(i)}} T^{(i)^{\dagger}}\right]\left(\mathbf{x}^{(j)}+\mathbf{x}^{(k)}\right), \quad i=1,2,3$.

In Eq. (24), the components of the matrices $H^{(i)}, U^{(i)}$, and $T^{(i)}$ are related to the spectroscopic amplitudes of the DRPA propagators, as explained in Appendix B. The $D^{(i)}$ 's are diagonal matrices containing the eigenvalues of the corresponding DRPA.

One can now define block-diagonal matrices $\mathbf{H}$, D, etc., that contain on the diagonal the matrices $H^{(i)}, D^{(i)}$, etc. These matrices act on the vectors $\mathbf{X}$ defined in Eq. (23). Using this notation one combines Eq. (24) as follows:

$$
\mathbf{X}=\left[\mathbf{H H}^{\dagger}+\mathbf{U} \frac{1}{\omega-\mathbf{D}} \mathbf{T}^{\dagger}\right] \mathbf{M X},
$$

where we have also introduced the matrix

$$
\mathbf{M}=\left[\begin{array}{lll} 
& I & I \\
I & & I \\
I & I &
\end{array}\right]
$$

that takes into account the proper mixing between the Faddeev components.

By introducing the vector

$$
\mathbf{Y} \equiv \frac{1}{\omega-\mathbf{D}} \mathbf{T}^{\dagger} \mathbf{M X}
$$

[which appears in Eq. (25)] and remembering that $\mathbf{D}$ is a diagonal matrix, it is possible to manipulate Eq. (25) into the usual form of an eigenvalue equation

$$
\omega \mathbf{X}=\mathbf{F X},
$$

where we have introduced the Faddeev Hamiltonian $\mathbf{F}$ [23], which is given by

$$
\mathbf{F}=\left[\mathbf{I}-\mathbf{H H}^{\dagger} \mathbf{M}\right]^{-1} \mathbf{U}\left\{\mathbf{T M}+\mathbf{D}\left(\mathbf{U}^{-1}\right)\left[\mathbf{I}-\mathbf{H} \mathbf{H}^{\dagger} \mathbf{M}\right]\right\} .
$$

The form (28) of the Faddeev eigenvalue equations is useful, since it reduces the problem to the diagonalization of a single (non-Hermitian) Hamiltonian. 
The Hamiltonian $\mathbf{F}$ can still correspond to a large matrix requiring a large amount of CPU time to diagonalize. As will be explained later in Sec. IV, about two-thirds of its solutions are trivial and without physical meaning. Thus, it is not necessary to diagonalize the full Faddeev Hamiltonian (29) but one can project it onto the space of physical solutions.

\section{Symmetry requirements and normalization conditions}

As a consequence of the Pauli exclusion principle, the spectroscopic amplitudes for the $2 \mathrm{p} 1 \mathrm{~h}$ motion have to be antisymmetric with respect to the exchange of the two particle indices. This statement applies to the full spectroscopic amplitudes (19) and (21) but not to the single Faddeev components, which have more complicated exchange properties. To exhibit the correct symmetry requirements for the Faddeev components, it is useful to introduce the following exchange operator, which works on the space (23) of the three $\mathbf{x}^{(i)}$ components:

$$
\mathbf{P}=\left[\begin{array}{ccc} 
& I^{e x} & \\
I^{e x} & & \\
& & I^{e x}
\end{array}\right]
$$

The form of matrix (30) takes into account that the component $x^{(1)}$ has to change into $x^{(2)}$ when the first two legs (i.e., the two particles) are exchanged. Since $\mathbf{P}$ is idempotent (i.e., $\mathbf{P}^{2}=\mathbf{P}$ ), it has only eigenvalues +1 and -1 and the respective eigenvectors are of the form

$$
\mathbf{X}_{-1}=\left[\begin{array}{c}
\mathbf{x}_{a} \\
-I^{e x} \mathbf{x}_{a} \\
\mathbf{x}_{b}-I^{e x} \mathbf{x}_{b}
\end{array}\right] \text { and } \mathbf{X}_{+1}=\left[\begin{array}{c}
\mathbf{x}_{a} \\
+I^{e x} \mathbf{x}_{a} \\
\mathbf{x}_{b}+I^{e x} \mathbf{x}_{b}
\end{array}\right]
$$

in which $\mathbf{x}_{a}$ and $\mathbf{x}_{b}$ are any two vectors. One easily recognizes that the three Faddeev components of $\mathbf{X}_{-1}$ and $\mathbf{X}_{+1}$ give rise to antisymmetric and symmetric spectroscopic amplitudes, respectively, when they are inserted in Eq. (21).

Using the symmetry properties of the interaction boxes (13) and the definition of $\mathbf{M}$ (26), one can show that $\mathbf{P}$ commutes with the matrix multiplying $\mathbf{X}$ in Eq. (25) and therefore with the Faddeev Hamiltonian (29). Thus, $\mathbf{P}$ and $\mathbf{F}$ must have a common set of eigenvalues. The relevant eigenvectors in the present case correspond to those involving $\mathbf{X}_{-1}$.

The normalization condition is derived as usual by considering the Lehmann representation (18) for the components $R_{\mu \nu \lambda, \alpha \beta \gamma}^{(i)}(\omega)[33,34]$. One can expand around a given pole $\varepsilon_{m}^{F d}$ and consider terms to order zero and then make use of the conjugate of the eigenvalue equation (24). The result is a condition for the $\mathbf{X}^{(i)}$ 's which only allows proper normalization for the antisymmetric component. These antisymmetric solutions $\mathbf{X}_{-1}$ satisfy the following condition:

$$
\mathbf{x}^{\dagger} \mathbf{x}-\sum_{i=1,2,3} \mathbf{y}^{(i)^{\dagger}} \mathbf{y}^{(i)}=2
$$

where $\mathbf{x}$ is the spectroscopic amplitude appearing in Eq. (21) and the factor of 2 appears because a sum over all indices of $x_{n_{1} n_{2} k}^{m}$ (20) is implied, which includes also the exchange terms. Eq. (32) differs from the usual normalization of a wave function for the fact that we have to subtract the additional terms

$$
\mathbf{y}^{(i)}=\left[V^{(i)} H^{(i)^{\dagger}}+J^{(i)} \frac{1}{\omega-D^{(i)}} T^{(i)^{\dagger}}\right]\left(\mathbf{x}^{(j)}+\mathbf{x}^{(k)}\right) \quad i=1,2,3 .
$$

These contributions correspond to the diagrams shown in Fig. 4 that have been discarded in the present expansion.

\section{TREATMENT OF SPURIOUS SOLUTIONS}

The Faddeev formalism is based on the introduction of different components $\mathbf{x}^{(i)}$, which belong to the same linear space of the total spectroscopic amplitude $\mathbf{x}$ (22). These components are the solutions of the Faddeev-eigenvalue equation (28), which is formulated in a larger space in terms of the vectors $\mathbf{X}$ containing all three $\mathbf{x}^{(i)}$. Only one-third of the solutions in this larger space have physical meaning while the others have to be discarded. One can clarify this problem by looking at how the complete spectroscopic amplitudes $\mathbf{x}$ are obtained from the components $\mathbf{x}^{(i)}$ through Eq. (21). Relevant details for treating this issue are discussed below.

The antisymmetric solutions of the Faddeev equations $\mathbf{X}_{-1}$ are determined from two independent vectors $\mathbf{x}_{a}$ and $\mathbf{x}_{b}$ as shown in Eq. (31). In particular, one has to specify both the symmetric and antisymmetric parts of the first vector $\left(\mathbf{x}_{a}\right)$ and only the antisymmetric part of the second $\left(\mathbf{x}_{b}\right)$. These solutions therefore belong to the space

$$
V_{F} \equiv V_{A} \otimes V_{A} \otimes V_{S} .
$$

The complete spectroscopic amplitudes $\mathbf{x}$ must also be antisymmetric under the exchange of the two particle indices, so they belong to $V_{A}$. Thus, Eq. (21) must be regarded as a projection from $V_{F}$ to the smaller space $V_{A}$ and therefore must have a nonvanishing kernel. We denote this kernel by $V_{S p}$ and refer to its vectors as spurious states, $\mathbf{Y}_{S p}$. Although these states satisfy the Pauli requirements, they do not yield any contribution to the full $2 \mathrm{p} 1 \mathrm{~h}$ propagator. We also consider the space $V_{P h}$, which is orthogonal to the kernel $V_{S p}$ and contains the antisymmetric states $\mathbf{Y}_{P h}$ that generate nonvanishing spectroscopic amplitudes $\mathbf{x}$. The vectors belonging to $V_{P h}$ produce contributions to the $2 \mathrm{p} 1 \mathrm{~h}$ propagator and therefore in the following they will be referred to as physical states. In Appendix C, explicit basis sets for the $V_{P h}$ and $V_{S p}$ spaces are given. Obviously, the combination of these two basis sets forms an orthogonal basis of $V_{F}$ and one has $V_{P h}$ $\equiv V_{A}$ and $V_{S p} \equiv V_{A} \otimes V_{S}$.

It must be stressed that in general the solutions of the Faddeev eigenvalue equation (28) do not automatically separate into the physical and spurious states just defined. Nevertheless, it is shown in Appendix $\mathrm{C}$ that the states of $V_{S p}$ are proper eigenstates of the Faddeev Hamiltonian for the expansion presented in this paper. This feature always occurs for the three-body problem but is not guaranteed when working 
with quasiparticle and quasihole excitations unless a proper set of diagrams is considered. When this condition is satisfied, there exists a set of spurious solutions of the Faddeev equation (28) that spans the space $V_{S p}$ completely. The projection of the Faddeev Hamiltonian (29) onto the physical and spurious subspaces $V_{P h}$ and $V_{S p}$ then takes the form

$$
\mathbf{F}=\left[\begin{array}{cc}
\langle P h|\mathbf{F}| P h\rangle & 0 \\
\langle S p|\mathbf{F}| P h\rangle & \langle S p|\mathbf{F}| S p\rangle
\end{array}\right] .
$$

It should be noted that the physical states $\mathbf{Y}_{P h}$, belonging to $V_{P h}$, differ from the spurious ones $\mathbf{Y}_{S p}\left(\in V_{S p}\right)$ not only because they give rise to physically meaningful spectroscopic amplitudes but also because they are not solutions of the Faddeev equations (28). In general, a physically meaningful eigenvector of (35), $\mathbf{X}_{\text {physical }}$, is a mixture of states belonging to both $V_{P h}$ and $V_{S p}$, due to the mixing term $\langle S p|\mathbf{F}| P h\rangle$. Thus,

$$
\mathbf{X}_{\text {physical }}=c_{1} \mathbf{Y}_{P h}+c_{2} \mathbf{Y}_{S p},
$$

where $c_{1}$ and $c_{2}$ are some constants. In other words, a spurious component $\mathbf{Y}_{S p}$ is also generated that will be automatically projected out when computing the spectroscopic amplitude $\mathbf{x}(21)$.

It is important to recognize that such spurious contributions are indeed needed since they account for the differences of the three Faddeev components (20). The relation between the usual Faddeev components for a given physical or spurious state can be inferred from the basis sets (C1). There it is shown that all the Faddeev components $\mathbf{x}^{(i)}$ of a state $\mathbf{Y}_{P h}$ or $\mathbf{Y}_{S p}$ are equal up to a sign. As a consequence, if a general solution is a pure physical state $\mathbf{Y}_{P h}$, all its Faddeev components cannot differ from each other in a significant way. Having a mixing between physical and spurious states allows the possibility of obtaining two independent Faddeev components. This result corresponds to the physical ingredients that involve identical ph phonons for the components $\mathbf{x}^{(1)}$ and $\mathbf{x}^{(2)}$ but a pp phonon for $\mathbf{x}^{(3)}$.

When all the Faddeev components are summed to generate the full $\mathbf{x}$ in Eq. (21), the contribution of the spurious states cancels out. Thus, for any nonspurious solution of the Faddeev equations, only the contribution from physical states $\mathbf{Y}_{P h}$ is needed to determine the $2 \mathrm{p} 1 \mathrm{~h}$ propagator. By looking at Eq. (35), it is easy to see that these contributions can be directly obtained by diagonalizing the upper-left block

$$
\omega_{m} \mathbf{Y}_{P h}^{m}=\langle P h|\mathbf{F}| P h\rangle \mathbf{Y}_{P h}^{m},
$$

where $m$ is used to label the solutions. The solutions of Eq. (37) are sufficient to determine the $2 \mathrm{p} 1 \mathrm{~h}$ propagator. For some applications one may need the individual components $\mathbf{x}^{(i)}$. In that case, the contribution from spurious states $\mathbf{Y}_{S p}$ can be determined by solving the remaining part of the Faddeev equations

$$
\omega_{m} \mathbf{Y}_{S p}^{m}=\langle S p|\mathbf{F}| P h\rangle \mathbf{Y}_{P h}^{m}+\langle S p|\mathbf{F}| S p\rangle \mathbf{Y}_{S p}^{m} .
$$

We note that if the upper-right block of Eq. (35) is not zero, a mixing between the $\mathbf{Y}_{P h}$ and $\mathbf{Y}_{S p}$ states occurs for all the eigenstates of the Faddeev Hamiltonian. In this situation, the spurious eigenvalues will differ from the unperturbed energies and all of the solutions of the Faddeev equations will contain a component $\mathbf{Y}_{P h}$. The Faddeev formalism would therefore become useless, since it would no longer be possible to discern between "good" and "bad" solutions. In Appendix C we show how the correct behavior of spurious solutions is related to the presence of backward-going contributions of the DRPA $\Gamma$ matrices (see Fig. 3). In case these diagrams are neglected, the spurious states $\mathbf{Y}_{S p}$ no longer diagonalize the Faddeev Hamiltonian. Such diagrams may give a small contribution to the description of low-lying states but they are essential to make the whole formalism presented here meaningful. As a general rule, when deriving expansions based on the Faddeev equations, it should be kept in mind that not all possible sets of diagrams can be effectively summed to all orders. Instead, one must first check the consistency of the set of diagrams with respect to the behavior of spurious solutions.

\section{SUMMARY AND CONCLUSIONS}

The present theoretical description of the distribution of spectroscopic strength at low energies lacks important ingredients for a successful comparison with experimental data. One of these ingredients is a proper description of the coupling of s.p. motion to low-lying collective modes that are present in the system. Recent calculations for ${ }^{16} \mathrm{O}$ [10], for example, only include a TDA description of these collective modes. A new method is proposed here to study the influence of pp and ph RPA correlations on the s.p. propagator for a system with a finite number of fermions. This method is formulated in the context of SCGF theory by evaluating the nucleon self-energy in terms of the $2 \mathrm{p} 1 \mathrm{~h}$ and $2 \mathrm{~h} 1 \mathrm{p}$ propagators. The description of the $2 \mathrm{p} 1 \mathrm{~h}$ (or $2 \mathrm{~h} 1 \mathrm{p}$ ) excitations has been studied by using the Faddeev formalism, which is usually applied to solve the three-body problem. The Faddeev formalism is necessary since we consider the collective pp and ph RPA phonons as the basic building blocks to describe the $2 \mathrm{p} 1 \mathrm{~h}$ motion.

The computational scheme presented here employs only two-time propagators, thus leading to a tractable set of equations. At the same time the contributions of $\mathrm{pp}$ and $\mathrm{ph}$ RPA phonons have been consistently summed to all orders, thereby including the physical effects that appear to be relevant for the study of the ${ }^{16} \mathrm{O}$ nucleus. Unlike previous calculations in which ph phonons have been included, the present formalism takes the Pauli exchange correlations properly into account up to the $2 \mathrm{p} 1 \mathrm{~h}$ level.

In deriving the set of Faddeev equations, a formulation has been chosen that involves only a single diagonalization for the $2 \mathrm{p} 1 \mathrm{~h}$ fragments. The appearance of spurious solutions has also been discussed in some detail, showing that the inclusion of the contribution of certain diagrams is necessary to separate such spurious solutions from the physically meaningful ones. When this separation occurs, it is straightforward to project out the physical eigenstates from the Fad- 
deev equations, thereby eliminating the spurious ones.

The Faddeev formalism has been used to include specific correlations corresponding to $\mathrm{pp}$ and ph phonons in a natural way. Extensions to the inclusion of more complicated excitations like the extended DRPA [30] can be obtained in a convenient way by starting from the formalism presented in Sec. II.

The formalism presented here appears practical for describing the spectroscopic strength in ${ }^{16} \mathrm{O}$ in a similar space as was employed in [10]. This implementation is currently in progress and will be reported elsewhere.

\section{ACKNOWLEDGMENTS}

This work was supported by the U.S. National Science Foundation under Grant No. PHY-9900713.

\section{APPENDIX A: INTERACTION BOXES}

The $\tilde{\Gamma}^{(p p)}$ matrix (14a) obtained by solving the DRPA equation has the following Lehmann representation:

$$
\begin{aligned}
\tilde{\Gamma}_{\mu \nu, \alpha \beta}^{(p p)}(\omega) & =V_{\mu \nu, \alpha \beta}+\sum_{n+} \frac{\left(\Delta_{\mu \nu}^{n+}\right)^{*} \Delta_{\alpha \beta}^{n+}}{\omega-\varepsilon_{n+}^{\Gamma+}+i \eta}-\sum_{k-} \frac{\Delta_{\mu \nu}^{k-}\left(\Delta_{\alpha \beta}^{k-}\right)^{*}}{\omega-\varepsilon_{k-}^{\Gamma-}-i \eta} \\
& \equiv V_{\mu \nu, \alpha \beta}+\Delta \tilde{\Gamma}_{\mu \nu, \alpha \beta}^{>}(\omega)+\Delta \tilde{\Gamma}_{\mu \nu, \alpha \beta}^{<}(\omega)
\end{aligned}
$$

in which $n+(k-)$ label the forward-going (backward-going) contributions. In obtaining the $\Gamma^{(3)}$ vertex given by Eq. (13b) we want to keep both the forward- and backward-going terms of Eq. (A1). This implies that all three diagrams of Fig. 3 are included. The main problem encountered when working with dressed propagators is that the contribution of these three diagrams do not factorize in an expression of the form $G^{0>} \Gamma G^{0>}$ when $G^{0>}$ is represented by a propagator of the form

$$
G_{\mu \nu \lambda, \alpha \beta \gamma}^{0>}(\omega)=\sum_{n_{1}, n_{2}, k} \frac{\left(\mathcal{X}_{\mu}^{n_{1}} \mathcal{X}_{\nu}^{n_{2}} \mathcal{Y}_{\lambda}^{k}\right)^{*} \mathcal{X}_{\alpha}^{n_{1}} \mathcal{X}_{\beta}^{n_{2}} \mathcal{Y}_{\gamma}^{k}}{\omega-\left(\varepsilon_{n_{1}}^{+}+\varepsilon_{n_{2}}^{+}-\varepsilon_{k}^{-}\right)+i \eta}
$$

This factorization cannot be made because of the implicit sums over the particle and hole excitation indices $\left(n, n^{\prime}, k\right)$ in Eq. (A2). For example, the hole label $k$ cannot change in Fig. $3[12,30,35]$.

This difficulty can be overcome with a slight reformulation of the problem. We no longer regard $\Gamma^{(3)}$ and $G^{0>}$ only as functions of the model space indices $(\alpha, \beta, \gamma)$, but instead assign an additional dependence on the particle and hole indices. Thus promoting the $\left(n, n^{\prime}, k\right)$ quantum numbers to external indices, the Lehmann representation of $G^{0>}$ (17) will contain at most one pole for every matrix element. As a consequence, all the components (10) appearing in the Faddeev equations have to be reformulated in the same way. The original propagators can then be retrieved at the end by summing the solutions over all the particle and hole fragments. With this procedure it becomes possible to write the sum of the three diagrams in Fig. 3 in terms of a matrix product of two $G^{0>}(17)$ propagators and the following vertex:

$$
\begin{aligned}
\Gamma_{\mu n_{\mu} \nu n_{\nu} \lambda k_{\lambda}, \alpha n_{\alpha} \beta n_{\beta} \gamma k_{\gamma}}^{(3)}(\omega)= & \frac{1}{2} \frac{\delta_{k_{\lambda}, k_{\gamma}}}{\sum_{\sigma} \mid \mathcal{Y}_{\sigma}^{\left.k_{\lambda}\right|^{2}}}\left\{V_{\mu \nu, \alpha \beta}+\sum_{n+} \frac{\left(\Delta_{\mu \nu}^{n+}\right)^{*} \Delta_{\alpha \beta}^{n+}}{\omega-\left(\varepsilon_{n+}^{\Gamma+}-\varepsilon_{k_{\lambda}}^{-}\right)+i \eta}\right. \\
& \left.+\sum_{k-} \frac{\left[\omega-\varepsilon_{n_{\mu}}^{+}-\varepsilon_{n_{\nu}}^{+}-\varepsilon_{n_{\alpha}}^{+}-\varepsilon_{n_{\beta}}^{+}+\varepsilon_{k_{\lambda}}^{-}+\varepsilon_{k-}^{\Gamma-}\right] \Delta_{\mu \nu}^{k-}\left(\Delta_{\alpha \beta}^{k-}\right)^{*}}{\left(\varepsilon_{k-}^{\Gamma-}-\varepsilon_{n_{\mu}}^{+}-\varepsilon_{n_{\nu}}^{+}\right)\left(\varepsilon_{k-}^{\Gamma-}-\varepsilon_{n_{\alpha}}^{+}-\varepsilon_{n_{\beta}}^{+}\right)}\right\},
\end{aligned}
$$

which corresponds to the expression for the pp interaction box (13b). With this prescription, we are able to write an expansion that sums diagrams like those of Fig 3. This is achieved at the cost of an increased size of the matrices to be dealt with. After further manipulation, it is possible to avoid this complication by dropping the dependence on the model space indices $(\alpha, \beta, \gamma)$, as explained in Sec. III B. The advantage of the present procedure lies in the possibility to diagonalize the Faddeev amplitudes in one step instead of solving the equations with energy-dependent vertex functions as discussed in Sec. III C. The expressions for the ph interaction boxes $\Gamma^{(1)}$ and $\Gamma^{(2)}$ are derived in a completely analogous way.

\section{APPENDIX B: DRESSED RPA EQUATIONS}

To clarify the notations used in the paper, we give here a brief overview of the DRPA equation for the pp interaction matrix. We also give the explicit expressions for the normalization and closure relations used in the development of the formalism.

The pp-DRPA equation is derived from Eq. (14a) by choosing $K_{\alpha \beta, \gamma \delta}^{(p p)}=V_{\alpha \beta, \gamma \delta}$ and is shown in Fig. 5. Using the Lehmann representation (A1) and extracting the poles $\varepsilon_{n+}^{\Gamma+}$ $\left(\varepsilon_{k-}^{\Gamma-}\right)$ from the DRPA equation, we get the usual eigenvalue problem 


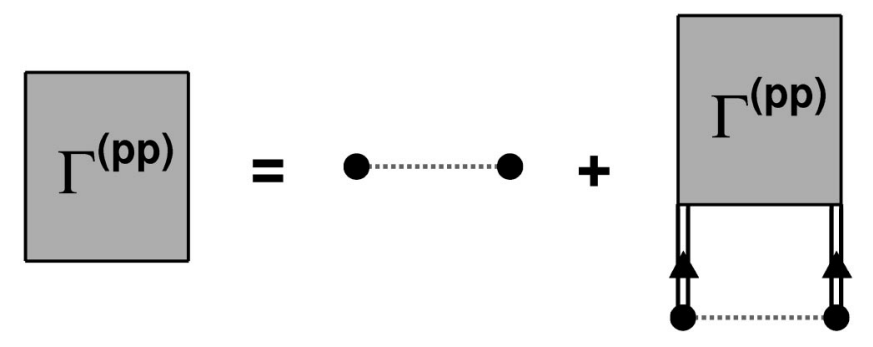

FIG. 5. DRPA equation for the $\widetilde{\Gamma}^{(p p)}$ matrix.

$$
\Delta_{\alpha \beta}^{n+(k-)}=\left.\Delta_{\gamma \delta}^{n+(k-)} g_{\gamma \delta, \epsilon \rho}^{(0)}(\omega) \frac{1}{2} V_{\epsilon \rho, \alpha \beta}\right|_{\omega=\varepsilon_{n+}^{\Gamma+}\left(\varepsilon_{k-}^{\Gamma-}\right)},
$$

where $n+(k-)$ refers to the forward-going (backwardgoing) solutions. It is useful to introduce the following notation, in analogy to the convention introduced in Eq. (20) for the Faddeev components:

$$
\begin{aligned}
& U_{n_{1}, n_{2}}^{n+}=\frac{\mathcal{X}_{\alpha}^{n_{1}} \mathcal{X}_{\beta}^{n_{2}}\left(\Delta_{\alpha \beta}^{n+}\right)^{*}}{\sqrt{2}\left(\varepsilon_{n+}^{\Gamma+}-\varepsilon_{n_{1}}^{+}-\varepsilon_{n_{2}}^{+}\right)}, \\
& H_{n_{1}, n_{2}}^{k-}=\frac{\mathcal{X}_{\alpha}^{n_{1}} \mathcal{X}_{\beta}^{n_{2}} \Delta_{\alpha \beta}^{k-}}{\sqrt{2}\left(\varepsilon_{k-}^{\Gamma-}-\varepsilon_{n_{1}}^{+}-\varepsilon_{n_{2}}^{+}\right)}, \\
& J_{k_{1}, k_{2}}^{n+}=\frac{\left(\mathcal{Y}_{\alpha}^{k_{1}} \mathcal{Y}_{\beta}^{k_{2}} \Delta_{\alpha \beta}^{n+}\right)^{*}}{\sqrt{2}\left(\varepsilon_{n+}^{\Gamma+}-\varepsilon_{k_{1}}^{-}-\varepsilon_{k_{2}}^{-}\right)}, \\
& V_{k_{1}, k_{2}}^{k-}=\frac{\left(\mathcal{Y}_{\alpha}^{k_{1}} \mathcal{Y}_{\beta}^{k_{2}}\right)^{*} \Delta_{\alpha \beta}^{k-}}{\sqrt{2}\left(\varepsilon_{k-}^{\Gamma-}-\varepsilon_{k_{1}}^{-}-\varepsilon_{k_{2}}^{-}\right)} .
\end{aligned}
$$

These represent the generalization to dressed propagators of the usual RPA components (the $\sqrt{2}$ has been inserted only in the pp case for convenience). In Eq. (B2) the quantities $\mathcal{X}$ $(\mathcal{Y})$ and $\varepsilon_{n}^{+}\left(\varepsilon_{k}^{-}\right)$represent the spectroscopic amplitudes and the poles of the forward-going (backward-going) part of the one-body propagator, while $\varepsilon_{n+(k-)}^{\Gamma+(-)}$ are the eigenvalues of the DRPA equation (B1).

The normalization condition for the DRPA solutions, given in terms of the components (B2), is the generalization of the normalization for the usual RPA [36] and can be put in matrix notation as

$$
\left[\begin{array}{ll}
U^{\dagger} & J^{\dagger} \\
H^{\dagger} & V^{\dagger}
\end{array}\right]\left[\begin{array}{ll}
I & \\
& -I
\end{array}\right]\left[\begin{array}{ll}
U & H \\
J & V
\end{array}\right]=\left[\begin{array}{ll}
I & \\
& -I
\end{array}\right],
$$

while the closure relations are given by

$$
\left[\begin{array}{ll}
U & H \\
J & V
\end{array}\right]\left[\begin{array}{ll}
I & \\
& -I
\end{array}\right]\left[\begin{array}{ll}
U^{\dagger} & J^{\dagger} \\
H^{\dagger} & V^{\dagger}
\end{array}\right]=\frac{1}{2}\left[\begin{array}{ll}
I-I^{e x} & \\
& I^{e x}-I
\end{array}\right],
$$

where $U, H, J$, and $V$ are the matrices containing the elements of Eq. (B2). In dealing with the formalism for the Faddeev equations, it is also useful to introduce the following two matrices:

$$
\begin{aligned}
& T_{n_{1}, n_{2}}^{n+}=\frac{1}{\sqrt{2}} \mathcal{X}_{\alpha}^{n_{1}} \mathcal{X}_{\beta}^{n_{2}}\left(\Delta_{\alpha \beta}^{n+}\right)^{*}, \\
& W_{k_{1}, k_{2}}^{k-}=\frac{1}{\sqrt{2}}\left(\mathcal{Y}_{\alpha}^{k_{1}} \mathcal{Y}_{\beta}^{k_{2}}\right)^{*} \Delta_{\alpha \beta}^{k-},
\end{aligned}
$$

which are trivially related to the components (B2a) and (B2d).

The matrix elements given in Eqs. (B2a)-(B2d) and (B5a),(B5b) correspond to the matrices $H^{(3)}, U^{(3)}$, and $T^{(3)}$ introduced after Eq. (24) for the 2p1h Faddeev expansion $(J$, $V$, and $W$ being the corresponding ones for the $2 \mathrm{~h} 1 \mathrm{p}$ expansion).

\section{APPENDIX C: PROPERTIES OF SPURIOUS STATES}

The set of solutions $\mathbf{X}_{-1}$ (31) that satisfy the Pauli requirements can be divided in to two subsets of physical $V_{P h}$ and spurious states $V_{S p}$. Orthogonal basis sets for these two spaces are given by

$\mathbf{Y}_{P h}=\left(\begin{array}{c}\mathbf{u}-\mathbf{u}^{e x} \\ \mathbf{u}-\mathbf{u}^{e x} \\ \mathbf{u}-\mathbf{u}^{e x}\end{array}\right) \in V_{P h} \quad$ and $\quad \mathbf{Y}_{S p}=\left(\begin{array}{c}-\mathbf{u} \\ +\mathbf{u}^{e x} \\ \mathbf{u}-\mathbf{u}^{e x}\end{array}\right) \in V_{S p}$,

where the $\mathbf{u}$ represent unit vectors that belong to the space (22). Their components are given by

$$
u_{n_{1} n_{2} k}=\delta_{n_{1}, n^{\prime}} \delta_{n_{2}, n^{\prime \prime}} \delta_{k, k^{\prime}}
$$

with $n^{\prime}, n^{\prime \prime}$, and $k^{\prime}$ fixed fragmentation indices that label all the possible $\mathbf{u}$. The vectors $\mathbf{u}^{e x}=I^{e x} \mathbf{u}$ are given by the exchange of the two particle indices $n_{1}$ and $n_{2}$. The physical states $\mathbf{Y}_{P h}$ are characterized by the fact that they do not produce vanishing spectroscopic amplitudes while the spurious states $\mathbf{Y}_{S p}$ do. Thus $V_{S p}$ represents the kernel of Eq. (21).

It is clear from Eq. $(\mathrm{C} 1)$ that the $\mathbf{Y}_{P h}$ states span a space equivalent to the space of antisymmetric vectors $\mathbf{x}$ (21), thus $V_{P h} \equiv V_{A}$. Analogously, the $\mathbf{Y}_{S p}$ states depend on both the symmetric and the antisymmetric parts of the $\mathbf{u}$ vectors, which implies $V_{S p} \equiv V_{A} \otimes V_{S}$. Therefore, the vectors (C1) form a basis for the full antisymmetric Faddeev space $V_{F}$ (34).

In general, the physical and spurious states $(\mathrm{C} 1)$ defined here are not solutions of the Faddeev equations (28), they simply define a basis over which these solutions can be expanded. Nevertheless, for both the normal three-body Faddeev equations and the expansion proposed in this paper, it can be seen that the spurious states $\mathbf{Y}_{S p}$ (and only those) diagonalize the Faddeev Hamiltonian. The eigenvalues correspond to the poles of the three freely propagating lines $\omega$ $=\varepsilon_{n^{\prime}}^{+}+\varepsilon_{n^{\prime \prime}}^{+}-\varepsilon_{k}^{-}(17)$. This feature serves as a sum rule on the solutions of the Faddeev equations and (unlike the case of three-body systems) is not always satisfied when applying the formalism to particle and hole excitations. Instead this property depends on the diagrams included in the expansion 
and a proper set of diagrams needs to be employed in order to apply the Faddeev formalism. For the particular Faddeev expansion described here, this constraint is achieved by including the backward-going terms of DRPA phonons in the $\Gamma^{(i)}$ matrices and by using the closure relations (B4), which turn out to play an important role. In the following, the proof that the $\mathbf{Y}_{S p}$ states of Eq. (C1) actually represent a set of spurious solutions of the present Faddeev formalism is outlined. This also clarifies the relationship between the correct behavior of the spurious solutions and the backward-going DRPA diagrams.

Consider a spurious state $\widetilde{\mathbf{Y}}_{S p}$ of the form $(\mathrm{C} 1)$, with $\tilde{\mathbf{u}}$ given by Eq. (C2) and eigenvalue $\widetilde{\omega}$. We now observe that the matrices $U$ (B2a) and $T$ (B5a) differ from each other only by an energy denominator. In particular, we have

$$
-\frac{1}{\tilde{\omega}-\mathbf{D}} \mathbf{T} \rightarrow \mathbf{U}
$$

were $\mathbf{U}, \mathbf{T}$, and $\mathbf{D}$ are defined in Sec. III. If the eigenvalue is given by $\widetilde{\omega}=\varepsilon_{n^{\prime}}^{+}+\varepsilon_{n^{\prime \prime}}^{+}-\varepsilon_{k}^{-}$, the equivalence of the left- and right-hand side holds only for the matrix elements having the same indices $\left(n^{\prime}, n^{\prime \prime}, k^{\prime}\right)$. Indeed, only in that case the denominator $\tilde{\omega}-\mathbf{D}$ will be equal to the one in Eq. (B2a). On the other hand, we see from Eq. (C2) that the components of $\tilde{\mathbf{u}}$ are nonzero only for the same indices. This allows the substitution of the $\rightarrow$ in Eq. (C3) by an equal sign when acting on the vector $\widetilde{\mathbf{Y}}_{S p}$. Substituting Eq. (C3) into Eq. (25) and using the closure relations of the DRPA, we obtain the equation

$$
\tilde{\mathbf{Y}}_{S p}=-\mathbf{M} \tilde{\mathbf{Y}}_{S p},
$$

which is valid only for the specific state $\widetilde{\mathbf{Y}}_{S p}$, labeled by the indices $\left(n^{\prime}, n^{\prime \prime}, k^{\prime}\right)$. The last equation is satisfied for a spurious state of the form (C1) but not for the corresponding physical state $\widetilde{\mathbf{Y}}_{P h}$. Thus, we have obtained a set of spurious solutions of the Faddeev equations that form an orthogonal basis of $V_{S p}$.

In this proof, we note that the closure relation (B4) can be applied to derive Eq. (C4) because of the presence of the backward-going term $\mathbf{H H}^{\dagger}$ in Eq. (25), which comes directly from the last diagram of Fig. 3.
[1] A.E.L. Dieperink and P. de Witt Huberts, Annu. Rev. Nucl. Part. Sci. 40, 239 (1990).

[2] I. Sick and P. de Witt Huberts, Comments Nucl. Part. Phys. 20, 177 (1991).

[3] L. Lapikás, Nucl. Phys. A553, 297c (1993).

[4] B.E. Vonderfecht, W.H. Dickhoff, A. Polls, and A. Ramos, Phys. Rev. C 44, R1265 (1991).

[5] H. Müther and W.H. Dickhoff, Phys. Rev. C 49, R17 (1994).

[6] M. Radici, S. Boffi, S.C. Pieper, and V.R. Pandharipande, Phys. Rev. C 50, 3010 (1994).

[7] G.A. Rijsdijk, K. Allaart, and W.H. Dickhoff, Nucl. Phys. A550, 159 (1992).

[8] M. Leuschner et al., Phys. Rev. C 49, 955 (1994).

[9] D. Van Neck, M. Waroquier, A.E.L. Dieperink, S.C. Pieper, and V.R. Pandharipande, Phys. Rev. C 57, 2308 (1998).

[10] W.J.W. Geurts, K. Allaart, W.H. Dickhoff, and H. Müther, Phys. Rev. C 53, 2207 (1996).

[11] P. Czerski, W.H. Dickhoff, A. Faessler, and H. Müther, Phys. Rev. C 33, 1753 (1986).

[12] J. Yuan, Ph.D. thesis, Washington University, St. Louis, 1994.

[13] W.H. Dickhoff, in Nuclear Methods and the Nuclear Equation of State, edited by M. Baldo (World Scientific, Singapore, 1999), p. 326.

[14] P. Schuck, F. Villars, and P. Ring, Nucl. Phys. A208, 302 (1973).

[15] G.A. Rijsdijk, W.J.W. Geurts, K. Allaart, and W.H. Dickhoff, Phys. Rev. C 53, 201 (1996).

[16] P. Danielewicz, Ann. Phys. (Leipzig) 197, 154 (1990).

[17] P. Danielewicz and P. Schuck, Nucl. Phys. A567, 78 (1994).

[18] A.D. Jackson, A. Lande, and R.A. Smith, Phys. Rep. 86, 55 (1982).
[19] L.D. Faddeev, Zh. Éksp. Teor. Fiz. 39, 1459 (1961) [Sov. Phys. JETP 12, 1014 (1961)].

[20] W. Glöckle, The Quantum Mechanical Few-Body Problem (Springer, Berlin, 1983).

[21] S.K. Adhikari and W. Glöckle, Phys. Rev. C 19, 616 (1979).

[22] J.W. Evans and D.K. Hoffman, J. Math. Phys. 22, 2858 (1981).

[23] P. Navrátil, B.R. Barrett, and W. Glöckle, Phys. Rev. C 59, 611 (1999).

[24] A. L. Fetter and J. D. Walecka, Quantum Theory of ManyParticle Physics (McGraw-Hill, New York, 1971).

[25] A.A. Abrikosov, L.P. Gorkov, and I.E. Dzyaloshinski, Methods of Quantum Field Theory in Statistical Physics (Dover, New York, 1975).

[26] P.C. Martin and J. Schwinger, Phys. Rev. 115, 1342 (1959).

[27] J. Winter, Nucl. Phys. A194, 535 (1972).

[28] C.J. Joachain, Quantum Collision Theory (North-Holland, Amsterdam, 1975).

[29] P. Schuck and S. Ethofer, Nucl. Phys. A212, 269 (1973).

[30] W.J.W. Geurts, K. Allaart, and W.H. Dickhoff, Phys. Rev. C 50, 514 (1994).

[31] S. Amari (private communication).

[32] J.P. Blaizot and G. Ripka, Quantum Theory of Finite Systems (MIT Press, Cambridge, MA, 1986).

[33] W. Hengeveld, W.H. Dickhoff, and K. Allaart, Nucl. Phys. A451, 269 (1986).

[34] S.D. Yang and T.T.S. Kuo, Nucl. Phys. A456, 413 (1986).

[35] W.J.W. Geurts, Ph.D. thesis, Free University, Amsterdam, 1996.

[36] P. Ring and P. Schuck, The Nuclear Many-Body Problem (Springer, New York, 1980). 